\title{
Soft Computing: Coping with Complexity
}

\author{
Enrique H. Ruspini \\ European Centre for Soft Computing \\ Edificio Científico Tecnológico, c/ Gonzalo Gutiérrez Quirós, s/n \\ 33600 Mieres, Asturias, Spain \\ E-mail: enrique.ruspini@softcomputing.es \\ Received: 01-12-2009 \\ Accepted: 28-05-2010
}

\begin{abstract}
While it has been correctly noted that the expression "soft computing" is employed in a variety of senses and characterizations to designate a wide array of technologies, we argue that the original motivation for the introduction of soft-computing methods was the need to cope with the complexity of existing mathematical and computational models of real-world systems. We review this essential motivation contending that other apparent distinguishing characteristics-such as the implicit character of some models-are either artifacts of specific treatments or temporary stages in their evolution towards more sophisticated technologies.
\end{abstract}

Keywords: Modeling, Complexity, Soft Computing, Imprecision, Uncertainty, Vagueness.

\section{Introduction}

The expression "soft computing" is primarily employed nowadays to designate a family of computational approaches to the modeling and analysis of real-world systems. As evidenced by the various contributions to this issue, their common characterization in terms of shared traits and properties is not easy. Efforts to trace the origin of qualifier by examining the bibliographical record are a bit disappointing. The term "soft" appears firstly to describe inconvenient characteristics of experimental data rather than to describe a particular class of mathematical and computational approaches. Even then, as Zadeh noted in $1981^{32}$, the meaning of the qualifier was not clear

The term soft data does not have a universally agreed upon meaning. Some researchers use it to characterize data that are imprecise or uncertain, while others attach the label "soft" to data whose credibility is open to question.

The introduction of artificial intelligence systems employing rule-based approaches ${ }^{8}$ —inspired primarily by applications of conventional two-valued logic - coupled with the development of the deductive machinery of fuzzy logic ${ }^{31}$ permitted the extension of rule-based ideas to schemes based on fuzzy-set theory and its successful application to many important applications, particularly in control systems. ${ }^{17,13,28}$ Concurrently with these advances, the need to represent and manipulate probabilistic uncertainty, handled by heuristics in early expert systems, ${ }^{12,27}$ resulted in a number of approaches based on conventional probability theory ${ }^{19}$ and its generalizations. ${ }^{26}$ These approaches were firstly thought as being unsound counterpartsdevised to deal with certain inconvenient features of 
data and knowledge — of the deductive procedures of classical logic; thus their description as being approximate-reasoning methods. The sound nature of these approaches, however, was made clear by their explanation in terms of formal logical models $21,23,25$ or by direct introduction of related methods using similar formalisms ${ }^{6}$ in the early 90 s.

At about that time, other approaches such as neural-network methods, evolutionary computation techniques, and genetic algorithms were increasingly applied to a variety of problems that had been previously intractable using existing formalisms. A more important development-at least from our task of characterizing the notion of soft computingwas the combined, or hybrid, application of these techniques together with fuzzy-set based methods. While the original motivation of these approaches had been the emulation of certain biological processes for computational purposes, their successful joint application to a variety of problems led to the need of differentiating them, as a whole, from more conventional treatments. This need brought a subtle change in the usage of the qualifier soft, whose scope changed from a description of the characteristics of the data being analyzed (i.e., soft-data analysis) to the analytical techniques themselves (i.e., soft data analysis). As has been noted in the lead article of this issue ${ }^{16}$ it was Zadeh ${ }^{34}$ who first employed the expression "soft computing" to designate this methodological ensemble. The alternative expression "computational intelligence" was also coined at about the same time to designate these methods ${ }^{\text {a }}$.

In this paper we argue that its original and continuing motivation of soft-computing methods is the need to cope with the complexity of models developed to understand real-world systems. Our discussion reviews this essential motivation contending that other apparent distinguishing characteristicssuch as the implicit character of some modelsare not distinguishing traits of soft-computing treatments.

\section{Systems and models}

To a very large extent, the misunderstandings regarding the nature of soft-computing methods can be traced to a confusion between the systems being analyzed and the models employed to perform that analysis, which ascribes to the former some of the distinguishing characteristics of the latter. This confusion is not unlike that equating the "inherent" complexity of problems - that is, the difficulty of solving a problem regardless of the treatment being employed - with that of the computational complexity of algorithms - that is, a quantification of the resources required by the algorithm as a function of the size of its input. ${ }^{1}$

In the context of systems theory, for example, it is customary to talk about linear systems and nonlinear systems although clearly the underlying notion of linearity describes a property of the model being employed, which may or may not adequately describe the actual behavior of the systems being studied. Thus systems are described as being stationary or non-stationary, ergodic and non-ergodic, and, not surprisingly, as hard or soft, while, in fact, these qualifications reflect properties of the mathematical and computational treatments being employed rather than essential characteristics of the actual systems.

This lack of distinction about systems and models has been particularly misleading when systems are treated using soft models or applying softcomputing techniques. Perhaps the most common mistake arising from this confusion is the notion that certain problems are intrinsically soft and, as such, demand treatment by soft-computing approaches. This misconception has led to needless controversy as to the existence of a fundamental need to employ soft computing methods as if there were measures of final recourse to be applied when everything else has failed. In its extreme form, this view has led some to argue, on a variety of grounds, that certain soft-computing methods, notably fuzzy logic, are unnecessary. ${ }^{5,10}$

The actual objects of study, that is, real-world

\footnotetext{
a The use of the expression "Computational Intelligence" stemmed from the internal need, within The Institute of Electrical and Electronics Engineers (IEEE), to describe the technical activities of those engaged in the study and application of these technologies with a better expression to characterize the broadening scope of activities of the IEEE Neural Networks Council
} 
systems. are neither soft nor hard, but may be, however, analyzed employing models based on the application of different methodologies. It makes sense to talk then about hard and soft models, or hard and soft approaches, but not of hard of soft systems. Furthermore, the fact that other approaches may conceivably or in fact, be applied is, in our view, irrelevant. Soft-computing methods provide an effective avenue, as evidenced by their success, to treat a wide scope of problems. This success owes much to their ability to deal with systems that had not been capable of effective prior treatment by other approaches but their applicability is not limited by availability of alternative treatments nor by any intrinsic characteristic of the systems being studied (e.g., lack of conventional models).

\section{The advent of soft computing}

In the 1960s, computing machines-employed before mainly for either accounting or numerical analysis applications-started to be applied in fields ranging from biology and medicine to economics, psychology, and the social sciences. The majority of the mathematical and computational structures introduce to formalize and represent the behavior of these systems did not attain, however, the basic objective that had traditionally led to the development of those models: the understanding of the behavior of the real-world systems being modeled.

As larger and more complex systems were being considered, models became themselves larger, more complex, and more difficult to analyze, turning often to be intractable. These constructs were unmanageable for a number of reasons. Even when the model was thoroughly specified in terms of wellknown mathematical constructs (e.g., differential equations), the number and interrelations between them was usually so large as to prevent either a mathematical or a numerical solution. In other instances the problems were complicated by the presence of structures not easily handled by existing analytical techniques (e.g., nonlinear relations, mixed discrete/continuous variable problems).

In many cases, the situation was complicated by lack of precise knowledge about the system being modeled: a state of affairs that was not helped by introduction of approaches relying on parameters (e.g., probability distributions) that were either not known or that increased, rather than reduced, the complexity of the model. Often it was also the case that requirements imposed upon solutions (e.g., precise optimization of a functional) were (and still are) beyond existing analytical and computational capabilities.

Simplification of models by suitable approximations has always been a favorite recourse of modelers. Linearization techniques or approximation of complex physical laws by simpler counterparts (e.g., "the perfect-gas law") have been, for example, standard equipment in the modeling toolbox. Complex models of large systems, however, were now often not amenable to such approximations. The frustration induced by this lack of tractability led, sometimes, to purported proofs of universal impossibility of solution of some problems often on the grounds of the inability to deal with certain inconvenient features of the model, usually confusing the actual system with the model (e.g., methodological difficulties encountered with a linear approximation were described as intrinsic, systemic, obstacles to any type of treatment). In many cases, oversimplification led to results of considerable formal beauty but, unfortunately, of little value to those seeking to understand the system being modeled.

It was in the context of these methodological difficulties that the notion of fuzzy set was introduced in $1965^{29}$ as

... a convenient point of departure for the construction of a conceptual framework which parallels in many respects the framework used in the case of ordinary sets, but is more general than the latter and, potentially, may prove to have a much wider scope of applicability particularly in the fields of pattern recognition and information processing.

The introduction of this key concept was motivated, however, by larger concerns than the mere representation of imprecision and vagueness as explicitly stated in a subsequent work: ${ }^{30}$ 
What we still lack, and lack rather acutely, are methods for dealing with systems that are too complex or too ill-defined to admit of precise analysis. Such systems pervade life sciences, social sciences, philosophy, economics, psychology and many other "soft" fields. Furthermore, they are encountered in what are normally regarded as "nonsoft" fields when the complexity of the system rules out the possibility of analyzing it by conventional mathematical means, whether with or without the aid of computers.

The reference to "soft" sciences reveals, as is the case with the previously mentioned allusion to soft data, the original intent to provide a new paradigm to cope with system complexity whether such complexity arose from model size or structure, vagueness, partial knowledge, or imprecision. This essential and motivating concern evolve into specific proposals for system analysis techniques in subsequent papers $^{31,32}$ leading to the introduction of the term "soft computing" in 1994. ${ }^{33}$

Note also that, as we have mentioned before, Zadeh's concerns about the need for new approaches to system analysis does not, at all, presume that his proposals should be considered to be a call for the replacement for existing methodologies; an assertion strongly stated in later works. ${ }^{35}$ As other developments would prove it was not necessary either for a problem to be tractable by other approaches to be successfully solved by application of fuzzy models. The inverse-pendulum problem-a well known paradigm of problems involving unstable systemsis a noteworthy example of the applicability of softcomputing methods ${ }^{3}$ in spite of availability of conventional models (i.e., a system of nonlinear differential equations) and alternative treatments. The application of soft-computing techniques to this problem (in this case fuzzy rules tuned by reinforcement learning techniques) further shows the simplic- ity of the approaches and the ability of the methodology to consider important variations of the original problem. ${ }^{\mathrm{b}}$

As this and many other examples show it is clear that the lack of an explicit model is not an inherent requirement for the application of soft-computing techniques. In many instances, as in applications of neural network techniques, the model is explicitly stated or learned, although its nature is quite different from that of conventional mathematical and statistical models. In other cases the model might be expressed by means of a complex computer program developed to facilitate the solution of a complex optimization problem by evolutionary-computation techniques. In the case of fuzzy models, the initial, and still preponderant, reliance on implicit models has led some to conclude that lack of explicit models is a characteristic trait of either fuzzy logic or soft computing methods. The growing literature on explicit fuzzy models and their analysis clearly shows otherwise. $^{2}$

\section{The nature of soft computing}

Having looked at the motivations for the introduction of fuzzy logic we turn now our attention to two rather important questions. The first of these questions is related to the basic premise underlying the models based on the theories of fuzzy sets and fuzzy logic. The second has to do with the nature of the methods that, beyond fuzzy logic, are currently part of the soft-computing toolbox.

We have already stated our belief that the major motivation leading to the theory of fuzzy sets was the need to deal with a class of problems that were not capable of being treated by existing methods. We turn our attention now to the primary reason for the success of fuzzy logic and argue that the basis for its success justifies extending the use of the qualifier "soft computing" to methodologies such as evolutionary and neural computation.

\footnotetext{
${ }^{b}$ In the case of the inverted-pendulum system it is important to note that soft-computing techniques have been able to treat important variations of the basic problem (i.e., stabilize the pendulum for small deviations around the equilibrium point) such as the consideration of multiple stages, the attainment of multiple goals (e.g., pendulum balancing and cart positioning), and the synthesis of controls that achieve stability from any initial system state.
} 


\subsection{A fundamental tradeoff}

While a major reason for the introduction of fuzzy logic was the modeling of systems that are not known in a precise manner, the basis for its success lies on the exploitation of those models to treat problems that are not easily solved employing conventional methodologies. The strength of the methodology lies on the modeler's willing to accept a key tradeoff: that of representational and analytical accuracy for an increase in the understandability of the system. Simply put, fuzzy models typically sacrifice precision for a intelligibility and analyzability. Quoting again Zadeh: ${ }^{33}$

In traditional-hard-computing, the prime desiderata are precision, certainty, and rigor. By contrast, the point of departure in soft computing is the thesis that precision and certainty carry a cost and that computation, reasoning, and decision making should exploitwherever possible the tolerance for imprecision and uncertainty.

While this type of epistemological bargain might seem reasonable to many scientists, its departure from what others consider to be an essential trait of serious science has led to considerable controversy about the technology from its very inception. This controversy has shown itself in the form of epistemological arguments about its necessity, ${ }^{10}$ proofs of internal inconsistence based on gross misunderstandings of its formal bases, ${ }^{7}$ or claims about the intrinsic inadequacy of approaches, other than probabilistic schemes, ${ }^{5,15}$ to represent and manipulate imprecise information. In extreme cases, fuzzy logic has been described as "the cocaine of science" or "pornography". 11

Leaving aside mercurial comments quoted without providing any form of argumentation, cogent or otherwise, we must simply note that these contentions have been properly, and abundantly, addressed in the literature by many, including this author. $^{22,23,24}$

The unfortunate error of confusing mathematical elegance with model applicability continues, however, to this day. Its clearest expression may be found in many works in the literature whose authors insist on using models that utterly fail to represent essential aspects of the modeled system and that produce precise, but misleading, results just for the sake of methodological elegance.

\subsection{Soft computing beyond fuzzy logic}

The description of biologically-inspired methodologies for the modeling and analysis of systems and for the learning of those models among "computational intelligence" approaches was the immediate consequence of the successful combination of these methods with fuzzy logic to treat a wide variety of problems. ${ }^{36}$ Although a clear definition of that expression has not been provided to date, ${ }^{20}$ the grouping of these approaches with fuzzy logic under a common rubric reflects the importance of their combined use in practice. Notwithstanding this historical fact it can be argued that neural networks and evolutionary computation/genetic algorithm-based methods are like fuzzy logic, successful modeling and analysis techniques that tradeoff certain properties of classical approaches in exchange for gains in problem tractability.

Neural-network ${ }^{9}$ approaches, which are characterized by their plasticity and learnability, are based on the application of models suggested by the structure of the nervous system. The acquired models, however, are usually hard to explain in terms of related knowledge while typically failing to provide insights into the structure of the modeled systems. As is the case with fuzzy logic their application, at least to date, requires exchanging certain analytical benefits for others.

Approaches such as evolutionary and genetic algorithms ${ }^{18}$ permit treatment of complex models by relaxation of certain formal requirements (e.g., the need to produce precise optimizing values of performance functionals). The solutions provided by these techniques cannot be guaranteed to be optimal (other than in a "long run," probabilistic sense) in the same sense that the output of a conventional optimization algorithm would be if it were feasible of application.

Probabilistic methods are often plagued themselves by their own complexity problems, which are a necessary consequence of the need to specify a 
weaker form of structural relation (i.e., probability distributions) in lieu of unknown set-theoretic relations. These approaches are sometimes, however, described as soft-computing methods because, in combination with neural networks, ${ }^{14}$ permit the acquisition of a powerful family of descriptive probabilistiic models. ${ }^{\mathrm{c}}$ Generalized probabilistic formulations ${ }^{26}$ exhibit also considerable tolerance to less than perfect knowledge.

\section{References}

1. S. Arora and B. Barak. Computational Complexity: A Modern Approach. Cambridge University Press, 2009.

2. R. Babuška. An overview of fuzzy modeling and model-based fuzzy control. In H. B. Verbruggen and R. Babuška, editors, Fuzzy Logic Control: Advances in Applications, volume 23 of World Scientific Series in Robotics and Intelligent Systems, pages 3-36. World Scientific, 1999.

3. H. R. Berenji. A reinforcement learning-based architecture for fuzzy logic control. International Journal of Approximate Reasoning, 6(2):267-292, 1992.

4. C. Borgelt, M. Steinbrecher, and R. Kruse. Graphical Models: Representations for Learning, Reasoning, and Data Mining. John Wiley and Sons, 2nd. edition, 2009.

5. P. Cheeseman. An inquiry into computational understanding. Computational Intelligence, 4:58-66, 1988.

6. D. Dubois, J. Lang, and H. Prade. Possibilistic logic. In D. M. Gabbay, C. J. Hogger, and J. A. Robinson, editors, Handbook of Logic in AI and Logic Programming, volume 3. Oxford University Press, 1994.

7. C. Elkan. The paradoxical success of fuzzy logic. IEEE Expert, 9:3-8, 1994.

8. E. A. Feigenbaum. The art of artificial intelligence: Themes and case studies of knowledge engineering. In IJCAI, pages 1014-1029, 1977.

9. E. Fiesler and R. Beale. Handbook of Neural Computation. Oxford University Press, 1996.

10. S. Haack. Do we need fuzzy logic? International Journal of Man-Machine Studies, 11:437-445, 1979.

11. S. Haack. Deviant logic, fuzzy logic: beyond the formalism. The University of Chicago Press, Chicago, IL, 1996.

12. P. E. Hart. Progress on a computer-based consultant. In Proc. International Joint Conference on Artificial Intelligence, volume 2, pages 831-841, 1975.

13. L. P. Holmblad and J.-J. Ostergaard. Control of a cement kiln by fuzzy logic. In M. M. Gupta and E. Sanchez, editors, Fuzzy Information and Decision Processes. North-Holland, 1982.

14. M. I. Jordan, editor. Learning in Graphical Models, Adaptive Computation and Machine Learning. MIT Press, Cambridge, Mass., 1999.

15. D. V. Lindley. Scoring rules and the inevitability of probability. Int. Stat. Rev., 50:1-26, 1982.

16. L. Magdalena. What is soft computing? revisiting possible answers. International Journal of Computational Intelligence Systems, 2010.

17. E. H. Mamdani and S. Assilian. An experiment in linguistic synthesis with a fuzzy logic controller. International Journal of Man-Machine Studies, 7(1):1-13, 1975.

18. Z. Michalewicz. Genetic Algorithms + Data Structures $=$ Evolution Programs. Springer-Verlag, 3rd. edition, 1996.

19. J. Pearl. Bayesian networks: A model of self-activated memory for evidential reasoning. In Proceedings of the 7th Conference of the Cognitive Science Society, pages 329-334, University of California, Irvine, CA., 1985.

20. I. J. Rudas and J. Fodor. Intelligent systems. International Journal of Computers, Communications, and Control, III:132-138, 2008.

21. E. H. Ruspini. The logical foundations of evidential reasoning. Technical Note 408, AI Center, SRI International, 333 Ravenswood Ave., Menlo Park, CA 94025, 1986.

22. E. H. Ruspini. Approximate reasoning: Past, present, future. Information Sciences, pages 297-317, 1991.

23. E. H. Ruspini. On the semantics of fuzzy logic. Int. J. of Approximate Reasoning, 5:45-88, 1991.

24. E. H. Ruspini. Responses to elkan (enrique h. ruspini). IEEE Expert, 4:32-33, 1994.

25. E. H. Ruspini. The semantics of approximate reasoning. In C. Chen, editor, Fuzzy Logic and Neural Network Handbook, pages 5.1-5.28. McGraw Hill, 1996.

26. G. Shafer. A Mathematical Theory of Evidence. Princeton University Press, 1976.

27. E. H. Shortliffe. Computer-Based Medical Consultations: MYCIN. American Elsevier, New York, N.Y., 1976.

28. M. Sugeno. Introductory survey of fuzzy control. Information Sciences, 36(1-2):59-83, 1985.

29. L. A. Zadeh. Fuzzy sets. Information and Control, 8:338-353, 1965.

30. L. A. Zadeh. Towards a theory of fuzzy systems. Technical report, NASA CR-1432, 1969.

31. L. A. Zadeh. Outline of a new approach to the analysis of complex systems and decision processes.

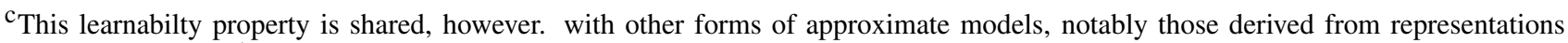
rooted on fuzzy sets 4
} 
IEEE Transactions On Systems Man And Cybernetics, SMC-3(1):28-44, 1973.

32. L. A. Zadeh. Possibility theory and soft data analysis. In L. Cobb and R. M. Thrall, editors, Mathematical Frontiers of the Social and Policy Sciences, pages 69129. Westview Press, 1981.

33. L. A. Zadeh. Fuzzy logic, neural networks, and soft computing. Communications of the ACM, 37(3):7784, March 1994.
34. L. A. Zadeh. Soft computing and fuzzy logic. IEEE Software, 11(6):48-56, November 1994.

35. L. A. Zadeh. Probability theory and fuzzy logic are complementary rather than competitive. Technometrics, 37(3):271-276, 1995.

36. J. M. Zurada, R. J. M. II, and C. J. Robinson, editors. Computational Intelligence: Imitating Life. IEEE Press, 1994. 